\title{
Keith Busby, Codex and Context. Reading Old French Verse Narrative in Manuscript
}

\section{Maria Colombo Timelli}

\section{(2) OpenEdition}

10 Journals

\section{Édition électronique}

URL : http://journals.openedition.org/studifrancesi/35636

DOI : $10.4000 /$ studifrancesi.35636

ISSN : 2421-5856

Éditeur

Rosenberg \& Sellier

\section{Édition imprimée}

Date de publication : 1 juillet 2005

Pagination : 125-126

ISSN : 0039-2944

\section{Référence électronique}

Maria Colombo Timelli, « Keith Busby, Codex and Context. Reading Old French Verse Narrative in

Manuscript », Studi Francesi [En ligne], 145 (XLIX | I) | 2005, mis en ligne le 30 novembre 2015, consulté

le 18 avril 2021. URL : http://journals.openedition.org/studifrancesi/35636 ; DOI : https://doi.org/

10.4000/studifrancesi.35636

Ce document a été généré automatiquement le 18 avril 2021.

\section{(c)}

Studi Francesi è distribuita con Licenza Creative Commons Attribuzione - Non commerciale - Non opere derivate 4.0 Internazionale. 


\title{
Keith Busby, Codex and Context. Reading Old French Verse Narrative in Manuscript
}

\author{
Maria Colombo Timelli
}

\section{RÉFÉRENCE}

KEITH BUSBY, Codex and Context. Reading Old French Verse Narrative in Manuscript, Amsterdam-New York NY, 2002 («Faux Titre», 221-222), 2 vol., pp. 941.

1 Cet imposant travail de Keith Busby s'appuie sur le constat préalable que la littérature médiévale ne peut se lire que dans les manuscrits qui l'ont transmise. Une telle affirmation, qui n'est pas nouvelle et qui peut paraître dans une certaine mesure paradoxale, ne saurait cependant être contestée par ceux qui ont eu entre les mains ne serait-ce qu'une seule fois - un de ces précieux témoins des siècles passés, et qui ont pu toucher sur pièces, par le simple maniement des feuillets et par la vision d'ensemble de la page, le fait que le texte médiéval et sa transmission manuscrite forment une unité cohérente et indivisible dont aucune édition critique ne saurait rendre pleinement compte. C'est donc à une série richissime de documents des XIII ${ }^{e}-\mathrm{XIV}^{\mathrm{e}}$ siècles (copies de textes en vers des $\mathrm{XII}^{\mathrm{e}}$ et XIII ${ }^{\mathrm{e}}$ ) que Busby se rapporte, et dont il reproduit un nombre remarquable de pages et de détails, pour que le lecteur d'aujourd'hui, spécialiste ou étudiant, puisse réfléchir sur cette dimension toute concrète des processus de l'écriture et de la lecture du Moyen Age qui risque à tout moment, sinon de se perdre, d'échapper au lecteur des éditions scientifiques modernes.

2 Sept chapitres examinent avec une grande clarté et beaucoup d'exemples les différents aspects de cette transmission. Le premier, Manufacture and Sale, présente - avec de nombreux exemples à l'appui - un panoramique du 'système' de la production des manuscrits aux siècles envisagés: composition des codices, lieux et modes de copie, professionnels et entreprises, possesseurs. La nature matérielle du support qui nous a 
transmis la littérature médiévale implique en effet la prise en compte de la complexité même de cette facture.

Dans le deuxième chapitre, Varieties of Scribal Behaviour, Keith Busby examine quelques cas d'espèces: des copies qui prouvent des attitudes particulières chez les copistes à l'égard du texte qu'ils transcrivent. Il désigne ainsi quelques cas-types: les scribes fournissant des «copies-miroirs», où l'effort est très visible de reproduire, en même temps que le texte, sa mise en page et son aspect extérieur (ce qui n'exclut quand même pas le phénomène des variantes linguistiques), le «wilful scribe», dont l'exemple majeur est celui de Guiot, nous donnant à lire des copies très personnelles des textes qu'il a réunis dans le célèbre ms. Paris, BNF fr. 794, et les "correctors and editors», corrigeant à l'envi leur copie ou collationnant plusieurs modèles. Dans tous ces cas, c'est une vision moins monolithique du travail de copie et de la 'variance' du texte médiéval qui doit s'imposer aux philologues et éditeurs de textes d'aujourd'hui.

Le chapitre suivant, Mise en texte, mise en page, and Reader Manipulation, étudie d'abord l'apparence générale du texte (présence / absence / fréquence des abréviations, séparation des mots / segments graphiques, ponctuation), puis son organisation à l'intérieur du codex (disposition du texte sur colonnes, tituli, incipit / explicit, présence et localisation des grandes capitales et des lettrines). L'analyse d'un nombre important de manuscrits de romans, chansons de geste, recueils, permet de reconnaître des systèmes d'écriture qui, sans être toujours cohérents et interprétables univoquement, ont très certainement orienté la réception et la lecture des textes.

5 Avec le quatrième chapitre, Text, Miniature, and Rubric, on aborde la question du rapport entre texte et image(s) dans les manuscrits illustrés de quatre compositions narratives: le Roman de Renart (pour lequel la division en branches constitue un élément majeur pour la localisation sinon pour le contenu des illustrations), le cycle de la Croisade (dont les manuscrits ne partagent pas, en gros, le programme iconographique), le Roman d'Alexandre et les textes rattachés (pour lesquels les éditions n'arrivent pas toujours à rendre compte de la variété des témoins), Perceval (les cinq manuscrits considérés ne présentant pas un véritable programme iconographique commun, sauf pour ce qui est d'une relative prédominance de Perceval par rapport à Gauvain) et Continuations (pour lesquelles, les manuscrits étant richement illustrés, est proposée une réflexion sélective). Dans les cas étudiés, Keith Busby remarque, d'une part, une très grande diversité typologique, d'autre part il souligne le caractère relativement tardif des copies illustrées, qui représentent une génération ultérieure dans la transmission des textes concernés, et il reconnaît enfin le passage graduel, dont l'illustration constitue un indice sinon une preuve positive, d'une littérature essentiellement orale à une pratique de lecture personnelle.

6 Dans sa réflexion sur la lecture «en contexte» des œuvres médiévales (ch. 5, Readings in Context), Keith Busby examine d'abord le cas des chansons de geste, et dresse une taxonomie des manuscrits des anciens textes épiques: des copies les plus anciennes du Roland aux manuscrits cycliques construits autour de Guillaume d'Orange; des remarques plus rapides concernent la tradition de la Geste des Lorrains, Renaut de Montauban, les œuvres d'Adenet le Roi. Viennent ensuite les romans arthuriens postérieurs à Chrétien de Troyes: une analyse serrée des textes et de leurs thématiques permet de reconnaître les raisons qui fondent certains recueils. Les manuscrits de fabliaux constituent un troisième volet: le nombre des textes réunis, leurs groupements, les rapports de sens offrent autant de pistes de recherche. Une telle 
lecture ne nie certes pas la nécessité d'éditions critiques sérieuses, mais révèle des relations entre les textes impossibles à déceler vraiment lorsque ceux-ci sont excisés de leur contexte de transmission. Busby aborde enfin la question des recueils construits autour d'un genre ou d'une thématique (les vies de saints, les lais) ou autour d'un auteur (Marie de France ou Chrétien de Troyes constituent deux cas emblématiques). Le sixième chapitre (The Geography of the Codex) est centré sur les lieux de production non pas des textes, mais des manuscrits. Keith Busby suit un parcours géographique qui s'appuie sur la chronologie des témoins conservés: domaine anglo-normand; régions du Nord-Est de la France; Lorraine et Bourgogne; Champagne, Île de France, Paris; Italie enfin (Busby exclut justement les œuvres en 'franco-italien' pour se concentrer uniquement sur les mss. de textes français exécutés en Italie). Il en arrive à conclure que le rôle joué par le centre de la Francophonie - Paris et Île de France - dans ce domaine a été moins important que celui de certaines régions périphériques.

Le dernier chapitre (Ownership) porte sur les anciens possesseurs. Il s'agit d'un essai de reconstruction courageux, tant par les lacunes dans la documentation (inventaires post mortem, catalogues de libraires, testaments), que par la difficulté de retrouver, déchiffrer et interpréter des marques de propriété éparses dans les manuscrits (feuilles de garde, marges etc.). Pour des raisons de complétude - mais aussi parce que les documents font cruellement défaut pour les premiers siècles du Moyen Age - l'enquête s'étend ici jusqu'au XVI ${ }^{e}$ siècle. La présentation suit une sorte de hiérarchie sociale: royauté et noblesse de sang, noblesse de robe, Parlement, bourgeoisie, institutions et ordres religieux; deux paragraphes à part sont consacrés respectivement à l'Italie (avec les inventaires des grandes familles aristocratiques de ce pays: Gonzaga, ViscontiSforza, Estensi, Savoie) et aux siècles postérieurs au Moyen Age (grande aristocratie bourguignonne du XV siècle, nobles et bourgeois du siècle suivant).

Comme le souligne Keith Busby dans ses notes conclusives, plusieurs compétences sont nécessairement mises en jeu dans ce retour aux manuscrits: en histoire, en codicologie et paléographie, en histoire de l'art notamment, encore plus qu'en littérature. Tous les philologues et éditeurs de textes ne sauraient maîtriser un tel bagage de connaissances à un même degré, et au fond cela ne pourrait honnêtement leur être demandé. Cependant, ce qui doit être désormais pris en compte, c'est l'impératif de restituer aux textes médiévaux leur matérialité, et de les resituer dans leur contexte de production, que la pratique de l'édition critique risque à tout moment de gommer.

10 Les annexes sont imposantes par leur richesse: bibliographie (pp. 817-877), index des manuscrits (pp. 879-891) et index analytique (pp. 893-941) constituent un complément indispensable pour un ouvrage qui a toute chance de devenir un répertoire de référence pour la connaissance des manuscrits de textes en vers du Moyen Age français. 